\title{
La importancia de la terapia ocupacional a través del cuidado en pacientes psiquiátricos
}

\author{
María Salgado Quijano,* Guadalupe Espinosa Sánchez*
}

\begin{abstract}
RESUMEN
Introducción: El enfermo neuropsiquiátrico requiere de una estancia hospitalaria prolongada por las condiciones de su patología, por lo que es importante considerar a la persona en su aspecto biológico, psicológico y social. La terapia ocupacional es una alternativa y, a su vez, una herramienta que se utiliza como apoyo para la rehabilitación de este tipo de enfermos; además contribuye a que la persona se mantenga activa. Objetivo: Fomentar la integración grupal y el desarrollo de sus capacidades para expresar su creatividad. Metodología: El profesional de enfermería interviene en este proceso de rehabilitación de la enfermedad a través de la incorporación de las actividades que se realizan en la terapia; reincorpora a la persona lo antes posible a su vida cotidiana, familiar y social. La terapia ocupacional es una herramienta terapéutica que le permite al enfermo desarrollar su habilidad mediante actividades encaminadas a responder a las necesidades de tratamiento de cada persona. En algunos casos se implementan estrategias que faciliten la actividad que se está llevando a cabo. Meyer concebía al hombre como un organismo que se mantiene y equilibra en el mundo real, actualizándose a través de una vida activa y participativa. Resultados: A lo largo de la historia de la profesión, la ocupación humana ha sido típicamente clasificada por los terapeutas ocupacionales en tres áreas: autocuidado, incorporación a la vida social y laboral, todas ellas actividades productivas; así también el juego y las actividades de esparcimiento. Conclusiones: La terapia ocupacional juega un rol importante en la recuperación del enfermo neuropsiquiátrico, busca que la persona sea productiva durante su estancia hospitalaria, contribuye a mejorar su condición de salud y lo reintegra a su ámbito social y familiar.
\end{abstract}

Palabras clave: Terapia ocupacional, enfermo neuropsiquiátrico, enfermería.

\section{The importance of occupational therapy by taking care in psychiatric patients}

\begin{abstract}
Introduction: The neuropsychiatric illness requires a prolonged hospital stay by the conditions of their disease so it is important to consider the person in their biological, psychological and social. Occupational therapy is an alternative and also a tool used as support for the rehabilitation of such patients, helps the person to stay active. Objective: Encourage group integration and the development of their ability to express their creativity. Methodology: The nurse involved in the rehabilitation process of the disease through the incorporation of the activities taking place in therapy, rejoins the person as soon as possible to your everyday life, family and social. Occupational therapy is a therapeutic tool that allows the patient to develop their skills with activities to meet the treatment needs of each person; in some cases implemented strategies to facilitate the activity being carried out. Meyer conceived to man as an organism that maintains and balances in the real
\end{abstract}

* Licenciada en Enfermería. Enfermera con Curso Postécnico de Psiquiatría.

Correspondencia: María Salgado Quijano, Insurgentes Sur 3877. Col. La Fama. Delegación Tlalpan, 14269, México, D.F. E-mail: msalgadoq@hotmail.com 
world, updated through an active and participatory. Results: Over the history of the profession, human occupation has been typically classified by occupational therapists in three areas: self-care, incorporating the social and working life, all of these productive activities, also play and leisure activities. Conclusions: Occupational therapy plays an important role in the recovery of neuropsychiatric illness, looking for a person to be productive during their hospital stay, helping to improve their health, and their social reintegration and family.

Key words: Occupational therapy, neuropsychiatric illness, nursing.

\section{MARCO CONCEPTUAL}

$\mathrm{M}$ eyer concebía al hombre "como un organismo que se mantiene y equilibra en el mundo real, actualizándose a través de una vida activa y participativa". A lo largo de la historia de la profesión, la ocupación humana ha sido típicamente clasificada por los terapeutas ocupacionales en tres áreas: autocuidado, incorporación a la vida social y laboral, todas ellas actividades productivas, además del juego y las actividades de esparcimiento.

La terapia ocupacional, según la Organización Mundial de la Salud (OMS), "es el conjunto de técnicas, métodos y actuaciones que, a través de actividades aplicadas con fines terapéuticos, previene y mantiene la salud, favorece la restauración de la función, suple los déficit invalidantes y valora los supuestos comportamentales y su significación profunda para conseguir las mayores independencia y reinserción posibles del individuo en todos sus aspectos: laboral, mental, físico y social". ${ }^{2}$

"La terapia ocupacional es el arte y la ciencia de dirigir la participación del hombre en tareas seleccionadas para restaurar, fortalecer y mejorar el desempeño, facilitar el aprendizaje de aquellas destrezas y funciones esenciales para la adaptación y productividad, disminuir o corregir patologías, promover y mantener la salud. Interesa fundamentalmente la capacidad a lo largo de la vida productiva, el dominio de sí mismo y el ambiente."’3

La terapia ocupacional, de acuerdo a Gómez-Tolón, es el arte y la ciencia de dirigir la respuesta del paciente en la realización de la actividad intencionada hacia los objetivos específicos previamente analizados y seleccionados en función de las necesidades del enfermo impedido y con fines de evaluación, prevención, adiestramiento, reeducación, tratamiento y reinserción tendientes a conseguir el máximo grado de autonomía. ${ }^{4}$

\section{OBJETIVOS}

General. Mejorar la calidad de vida consiguiendo el máximo nivel de autonomía e integración del enfermo neuropsiquiátrico a través de la terapia ocupacional para lograr su independencia psicológica y social y con la finalidad de reintegrarlo a su ámbito social y familiar.
Específico. Mejorar, desarrollar, mantener, y/o recuperar el desempeño de las funciones motoras del enfermo para minimizar, de ser posible, su deterioro.

\section{ANTECEDENTES}

El uso terapéutico de la actividad y el movimiento ha sido apreciado desde el inicio de las civilizaciones más primitivas. Desde el 2,600 a.C., en China, se pensaba que la enfermedad era generada por la inactividad orgánica y se utilizaba el entrenamiento físico mediante una serie de ejercicios gimnásticos, similares a los que hoy se conocen como Tai-Chi, para promover la salud, e incluso, según sus creencias, para asegurar la inmortalidad.

En la Grecia clásica, Esculapio fundó el hospital de la Ciudad de Pérgamo en el año 600 a.C. Aquí se trataba de aliviar el delirium con el empleo de canciones, música, literatura y farsas. Años después (entre el 600 a.C. y el 200 d.C.) Pitágoras, Tales de Mileto y Orfeo utilizaron la música como remedio para distintos males del cuerpo y la mente. Hipócrates, quien resaltaba la relación cuerpo-mente (mente sana en cuerpo sano) recomendó la lucha libre, la lectura y el trabajo para mantener y mejorar el estado de salud de los individuos.

Para aliviar "las mentes trastornadas", Cornelio Celso (14-37 d.C.) recomendaba la música, la conversación, la lectura, el ejercicio hasta la fatiga, los viajes y hasta un cambio de escena; para el mantenimiento de la salud, sus recomendaciones se dirigían hasta la navegación, el manejo de las armas y actividades como el juego de pelota, saltos, carreras y paseos. Para los problemas del estómago aconsejaba realizar lecturas en voz alta, así como otras actividades para diferentes dolencias. Las ocupaciones en esa época deberían ajustarse al temperamento de cada persona. Así mismo, Séneca (55 a.C. y 39 d.C.) aludía que el trabajo era remedio de cualquier agitación mental.

Durante la Edad Media, apenas se pudo desarrollar la idea del uso de la ocupación con fines terapéuticos, por lo que hubo que esperar hasta el renacimiento de todas las ciencias. ${ }^{5}$ A lo largo de los siglos XVIII y XIX aparecieron gradualmente los primeros patrones especializados sobre terapia ocupacional. 
En este orden de ideas, Philippe Pinel, médico francés especializado en el tratamiento de las enfermedades mentales, prescribió en 1801 ejercicios físicos y ocupaciones manuales para asegurar una buena moral y disciplina entre los pacientes psiquiátricos.

Resulta oportuno destacar que Benjamín Rush fue el primer médico que empleó el concepto de tratamiento moral y ocupación en los Estados Unidos; es evidente, entonces, que a él se le considera el padre de la psiquiatría norteamericana; sostenía que la locura era una enfermedad del cerebro y no una enfermedad del cuerpo. Consideraba que el hombre estaba hecho para ser activo, por lo que recomendaba el ejercicio, el trabajo y la música como parte del tratamiento de las enfermedades psiquiátricas. ${ }^{6}$

Cabe destacar que Susan E. Tracy fue la primera enfermera que creó el término enfermería ocupacional para el tratamiento de inválidos en 1905; ella impartió el primer curso de terapia ocupacional destinado a sus alumnas de enfermería en Boston, durante 1906; además publicó el primer libro titulado Ocupación para Inválidos (Studies in Invalid Occupations) en 1910, el cual es considerado el primer manual sobre terapia ocupacional. Éste señala la evaluación del paciente mediante la actividad ocupacional, es decir, analiza lo que hacen los enfermos para determinar sus limitaciones.

Cabe agregar que el médico Herbert J. Hall prescribía la ocupación para sus pacientes (lo que él denominaba cura de trabajo) como un tipo de medicina para dirigir el interés y regular la vida. En 1908 inició un programa de formación (inicialmente para enfermeras y trabajadoras sociales) que se basaba en el trabajo como tratamiento.

A partir de los años cincuenta, con la epidemia de poliomielitis y las secuelas de la parálisis cerebral, los terapeutas ocupacionales comenzaron a trabajar en el ámbito del neurodesarrollo y trataron también a la población infantil. En ese mismo sentido, Adolf Meyer, psiquiatra suizo, desarrolló sus ideas acerca de las causas y tratamientos de las enfermedades mentales; consideraba que los trastornos mentales eran resultado de hábitos o comportamientos desorganizados que daban lugar a problemas en el transcurso de la vida. Pensaba que una persona debía organizar su vida diaria en periodos de trabajo y juego y descanso, pues de esta forma lograría un equilibrio y armonía con la naturaleza. En 1921 presentó el primer modelo organizado de terapia ocupacional que muestra la importancia del ritmo de las actividades de una persona; afirmaba que un cambio de ritmo en la vida es lo que desencadena una patología. En efecto, Meyer consideraba que un deterioro mental acarrea un deterioro físico y viceversa. ${ }^{8}$ En ese orden de ideas, en España, en 1980, Gary Kielhofner y Mary Reilly estudiaron juntas la carrera; ambas empezaron a hacer su tesis en la cual establecieron un modelo nuevo de tratamiento conocido como de ocupación humana (MOHO). En 1995 se propone un modelo social de salud a partir de la rehabilitación médica y la propuesta de un paradigma de la terapia ocupacional; éste se basa en la naturaleza ocupacional del ser humano como determinante del bienestar. La propuesta se implementa en áreas de especialidad psiquiatría. ${ }^{9}$

En México, la fundación Teletón ha manifestado gran interés por llevar a cabo una profesionalización en el ámbito de la terapia ocupacional, iniciando su gestión en el año 2000 en el campo de la educación profesional con la creación del Instituto Teletón de Estudios Superiores en Rehabilitación (ITESUR), el cual nació con el objetivo de formar profesionales en rehabilitación necesarios para atender las demandas de las personas con discapacidad en nuestro país.

Ese mismo año, Fundación Teletón firmó un convenio de colaboración con la Universidad Autónoma del Estado de México para la apertura de las licenciatura en terapia física y terapia ocupacional; esta última se creó en el año 2012 como la licenciatura en terapia, avalada por la Secretaría de Educación Pública, la cual tiene como objetivo formar profesionales en terapia ocupacional que sean capaces de prestar servicios a personas o poblaciones utilizando herramientas, estrategias y modelos propios de la disciplina con el fin de desarrollar, mantener y restaurar la ocupación y la capacidad funcional al máximo durante toda la vida, dentro de la esfera del bienestar humano, con capacidad reflexiva, dinámica, actitud de humanismo y ética profesional, así como fomentar la autonomía del profesional convertido en terapeuta ocupacional para que integre sus conocimientos, habilidades y destrezas y realice sus funciones con calidad, eficiencia y pertinencia, y con base en principios éticos. ${ }^{10}$ El interés por una rehabilitación y desarrollo integral del enfermo mental ha permitido la incorporación de la terapia ocupacional como una disciplina de la salud que desarrolla todas sus habilidades en función de las necesidades, contribuyendo con esto a la recuperación psicosocial de la persona. El éxito en la recuperación psicosocial funcional y la readecuación de comportamientos en las personas que además del tratamiento médico (farmacológico) son incorporadas a un programa de utilización sana del tiempo libre, constituye el fundamento gestor de la terapia ocupacional como un quehacer científico en pro de la salud. ${ }^{11}$

\section{Implementación de la terapia ocupacional dentro del Instituto Nacional de Neurología y Neurocirugía}

\section{Aspectos históricos}

En 1964 se inaugura el Instituto Nacional de Neurología y Neurocirugía por el Dr. Manuel Velazco Suárez (INNN). 
En el terreno destinado para la construcción del Instituto ya existía la granja de recuperación para enfermos mentales y la Escuela para Adolescentes Deficientes Mentales "Bernardino Álvarez", las cuales quedaron integradas al propio Instituto.

Recién creada la Dirección General de Neurología, Salud Mental y Rehabilitación dentro de la SSA, al comienzo del ejercicio presidencial del Lic. Adolfo López Mateos, el Dr. Manuel Velazco Suárez tuvo la acertada idea de brindar atención a enfermos mentales crónicos capacitándolos para desarrollar actividades con fines terapéuticos; de esta manera se acondicionó un espacio. La granja de recuperación para enfermos mentales fue acondicionada para la atención de 80 enfermos masculinos, los cuales eran capacitados para realizar actividades de acuerdo a sus necesidades con fines terapéuticos; las actividades que desempeñaban eran diversas; una de ellas, la jardinería. En esta actividad se les capacitaba para la siembra y cultivo de hortalizas como lechuga, papas, col, entre otras; también se implementaron talleres de manualidades. Esta granja fue la primera en el D.F. y estuvo funcionando hasta 1972 cuando los enfermos fueron trasladados a otras granjas similares.

La Escuela para Adolecentes deficientes mentales "Bernardino Álvarez" empezó a funcionar en el año 1960 como dependencia de la Dirección General de Neurología, Salud Mental y Rehabilitación; a partir de 1964 se integró como Unidad Psicopedagógica del Instituto.

La capacidad de la escuela era para 100 alumnos cuyas edades comprendían de 12 a 20 años. La selección de los alumnos se basaba en estudios psicológicos para situarlos en el campo de trabajo más apropiado a sus condiciones mentales y de aptitud para el trabajo manual.

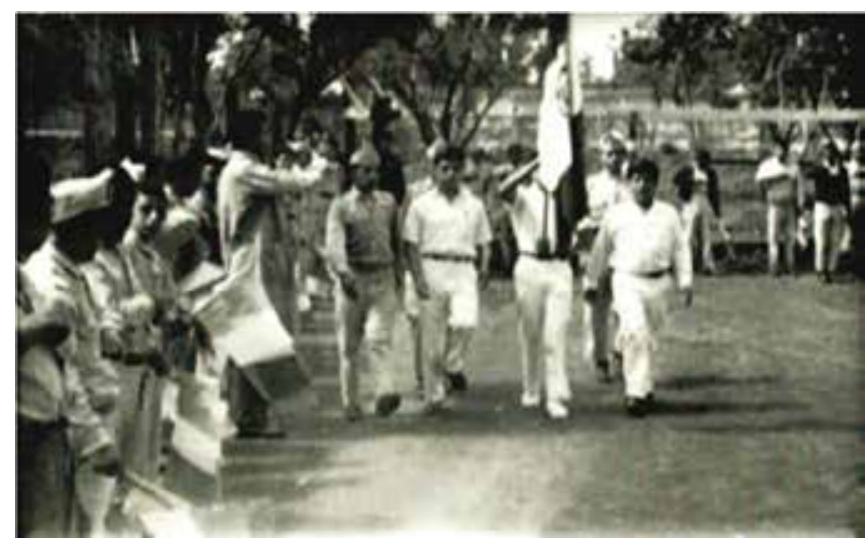

Figura 1. Honores a la bandera por los alumnos de la Escuela para Adolescentes Deficientes Mentales «Bernardino Álvarez», atendidos en el INNN, año de 1964. Fuente: Escobedo-Ríos, Rabadán-Quezada R. Instituto Nacional de Neurología y Neurocirugía, M. Editorial Progreso. México, 1974.
El programa de la escuela reunía dos objetivos fundamentales para los alumnos: a) ortopedia mental y b) habilitación en trabajo de talleres y actividades diarias. Para ello cuenta con dos grupos escolares y talleres de carpintería, encuadernación, talabartería, alfarería y cestería de mimbre; además se llevaban a cabo otras actividades como horticultura, deportes y honores a la bandera. Con estas actividades se pretendía que el débil mental llegara a ser un hombre útil.

\section{La realización de honores a la bandera}

En la Escuela para Adolescentes Deficientes Mentales "Bernardino Álvarez" el niño, al igual que el adulto, son un ser indivisible psíquica, bilógica y socialmente, pero con características especiales en cuanto al tipo de enfermedades; además, siendo susceptibles a las agresiones del medio ambiente aun antes de nacer y sabiendo que las alteraciones de tipo genético afectan frecuentemente al sistema nervioso, se creó el Servicio de Neuropediatría donde la intervención conjunta de especialistas de neurología, neurocirugía y psiquiatría puede afrontar el problema médico.

Con referencia a lo antes mencionado, también se vieron en la necesidad de crear la Clínica Psicológica y Terapéutica de Lenguaje para realizar estudios de inteligencia, personalidad, nivel de madurez, exploración de funciones lingüísticas, índice de velocidad y regularidad en el aprendizaje; memoria, atención e índice de fijación de la memoria, pruebas de lateralización cerebral, entre otros.

A su vez, se contaba con un salón de terapia ocupacional para los niños, en el cual se les enseñaban algunas actividades manuales y juegos de mesa tomando en cuenta su edad y estado de salud. ${ }^{12}$

En 1983 se creó el Servicio de Psiquiatría con capacidad para 41 camas, donde se reciben pacientes de ambos sexos

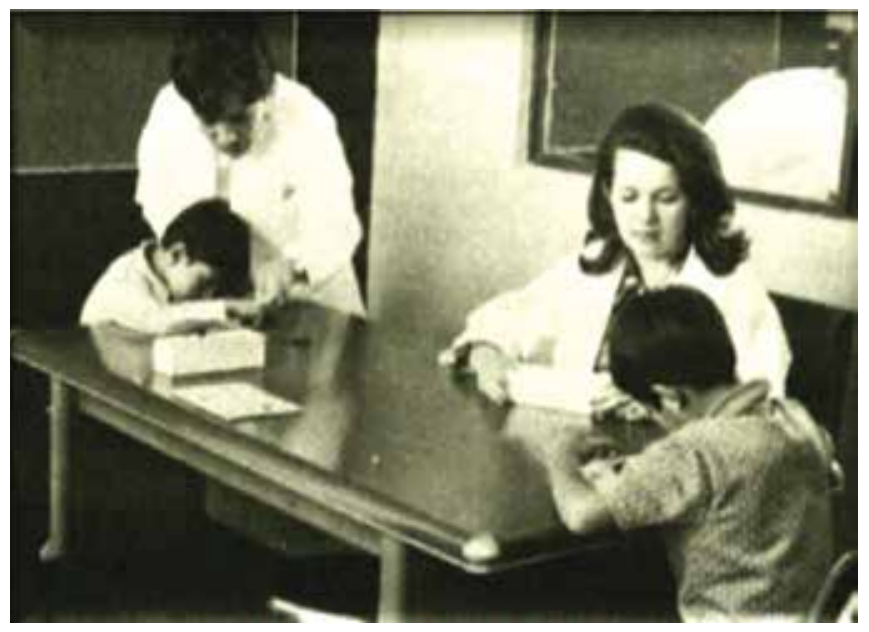

Figura 2. Sala para aplicación de pruebas. México, 1974. 


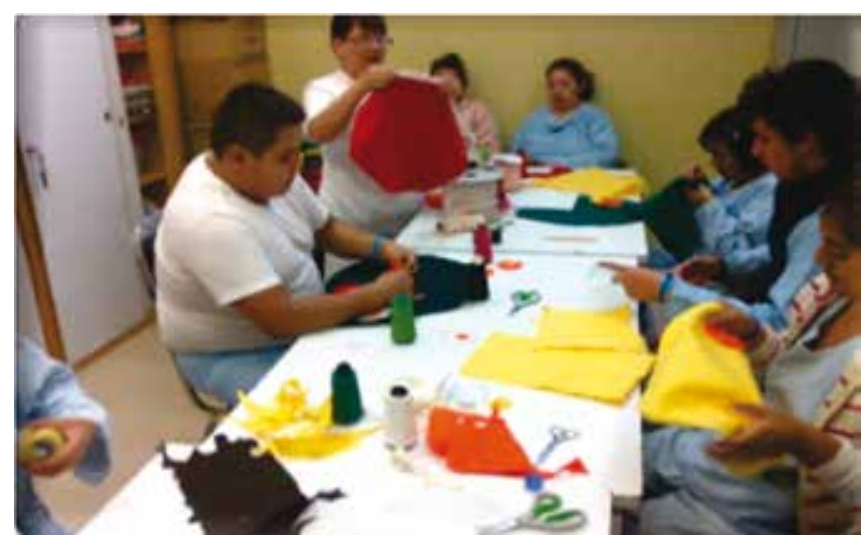

Figura 3. Sala de terapia ocupacional donde se llevan a cabo trabajos manuales. Año de 2013.

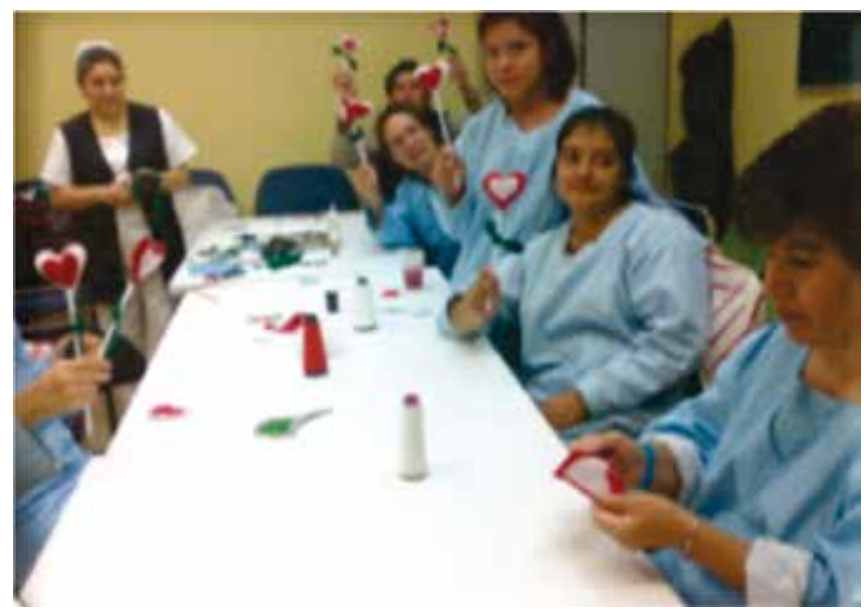

Figura 4. Terapia ocupacional: actividades con tema. 14 de febrero de 2013.

con padecimientos de evolución aguda y crónica con fines asistenciales, con objetivos de enseñanza e investigación. En esta área también se implementaban actividades manuales, las cuales se llevaban a cabo por dos terapistas ocupacionales que se hacían cargo de estas actividades con los pacientes psiquiátricos. Cuando las terapistas se jubilan, esta actividad se lleva a cabo por el profesional de enfermería realizando actividades recreativas y deportivas además de la terapia ocupacional que consiste en diversas actividades manuales. Actualmente, en el área de Neuropsiquiatría se atienden adultos con padecimientos neuropsiquiátricos agudos, a los cuales se les brinda atención de enfermería con calidad y calidez para que su estancia hospitalaria sea más confortable; para esto, el servicio cuenta con un salón donde se realiza terapia ocupacional, en el cual se desarrollan actividades manuales que los enfermos llevan a cabo bajo la supervisión de enfermería de lunes a viernes en el turno matutino; y martes, miércoles y viernes en el turno vesper-
Cuadro I. Fechas programadas para terapias ocupacionales.

\begin{tabular}{ll}
\hline Fecha & Conmemoración \\
\hline 6 de enero & Día de los reyes magos y la enfermera \\
5 de febrero & Constitución Política de México \\
14 de febrero & Día del amor y la amistad \\
24 de febrero & Día de la bandera \\
21 de marzo & Natalicio de Benito Juárez e inicio de la primavera \\
30 de abril & Día del niño \\
10 de mayo & Día del trabajo \\
5 de mayo & Batalla de Puebla \\
10 de mayo & Día de las madres \\
Tercer domingo de junio & Día del padre \\
16 de septiembre & Independencia de México \\
12 de octubre & Día de la raza \\
2 de noviembre & Día de muertos \\
20 de noviembre & Revolución Mexicana \\
25 de diciembre & Navidad \\
\hline
\end{tabular}

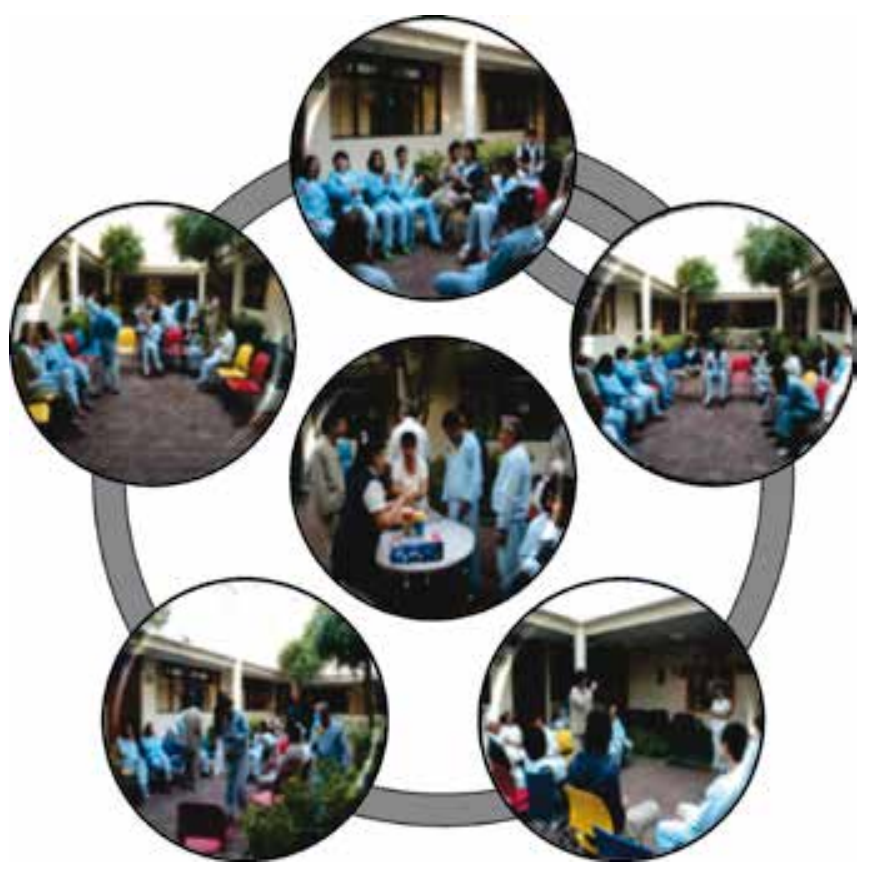

Figura 5. Imágenes de Terapia de Remotivación.

tino. Quien se encarga de solicitar el material para la terapia ocupacional es la supervisora del turno matutino con las damas voluntarias.

En el marco de las consideraciones anteriores, el profesional de enfermería ha buscado estrategias individuales y grupales que tienen que ver con la Remotivación y Grupo de Remotivación; por ello se precisan ambos términos.

Remotivación. "Es la utilización de técnicas especiales que estimulan a los pacientes para que se motiven a aprender e interaccionar." 13

Grupo de Remotivación. "Grupo terapéutico que se organiza con el propósito de estimular el interés, el conocimiento 
y la comunicación de los pacientes mentales abandonados e ingresados en instituciones." 14

En las remotivaciones se reúne a todos los enfermos con la finalidad de que se integren y participen; en primera instancia, cada uno se presenta diciendo su nombre, a qué se dedica y de dónde es... con la finalidad de que todos se conozcan y se establezca un ambiente cordial; así se va desarrollando el tema.

Se les dan tarjetas a los enfermos que contienen información importante acerca de la conmemoración alusiva al mes para que ellos lean el material y den su punto de vista acerca del tema que se esté desarrollando en ese momento; procurando que exista una interacción grupal, al término de esta remotivación se sociabiliza con los enfermos y se ofrece un ambigú; también se implementa otra técnica a través de la musicoterapia, amenizando el evento e integrando a los enfermos para que participen con baile y algunos con cantos. Con esta terapia de remotivación se pretende contribuir paulatinamente a la recuperación del enfermo neuropsiquiátrico.

\section{CONCLUSIONES}

Actualmente, los enfermos demandan la actividad que se realiza en la terapia ocupacional ya que ésta contribuye a restaurar la función mental del enfermo manteniéndolo ocupado.

Se ha observado que la terapia ocupacional es una herramienta terapéutica que beneficia al enfermo neuropsiquiátrico en sus distintas patologías; a los enfermos con depresión mayor la terapia ocupacional los ayuda a superar más pronto sus cuadros depresivos; a los que tienen trastornos de ansiedad la actividad los ayuda a canalizar su angustia; a los enfermos con psicosis y esquizofrenia la terapia ocupacional les favorece ya que al mantenerse activos su mente está concentrada en la actividad y esto los ayuda a ubicarse un poco en su espacio real. Estas actividades, realizadas en conjunto, contribuyen a que su estancia hospitalaria sea menor y de máximo beneficio.

Otras situaciones que han ayudado al enfermo neuropsiquiátrico son las remotivaciones grupales, en las cuales los beneficios que éstas proporcionan al enfermo contribuyen a ubicarlo en tiempo, lugar y espacio, además los motivan a la convivencia en grupo para integrarlos a la vida familiar y social.

Cabe destacar que esta actividad la lleva a cabo de manera programada y organizada el profesional de enfermería en el turno matutino y vespertino bajo sus propios recursos.

\section{BIBLIOGRAFÍA}

1. Beaudry de I, Portal de Terapia Ocupacional Pediátrica, OMS, disponible en cytycoyoacan2.com, consultado el 20 de febrero del 2013 a las 13:49 pm.

2. Diccionario Mosby, Medicina, Enfermería y Ciencias de la Salud, ediciones Hans Hancourt S.A. 2009.

3. Op.cit. Diccionario Mosby.

4. Escobedo F, Rabadán R, Instituto Nacional de Neurología y Neurocirugía. Editorial Progreso, 1974, México D.F., pp. 14-18.

5. Op.cit. Escobedo F. p. 83.

6. Hopkins H, Smith H. Terapia Ocupacional, 2001, octava edición, editorial Médica Panamericana S.A. México, D.F. p. 4.

7. Instituto Nacional de Neurología, Diez años de servicio, 1974, Secretaria de Salubridad y Asistencia, editores: Dr. Escobedo R. Francisco, Dr Rabadán Q. Ramiro. Editorial Progreso, S.A.

8. Polonio B, Durante P, Noya B. Conceptos Fundamentales de la Terapia Ocupacional. $1^{a}$ ed. Madrid, España. 2001, p. 41.

9. Op.cit. Polonio B. pp. 1-2.

10. Rincón E, Sánchez M, Ramos R, Terapia ocupacional. Intervención en distintas patologías. Ed. Formación. Alcalá España, 2007. p. 17.

11. Ibídem. Rincón. p. 19.

12. Ibídem. Rincón, p. 20.

13. Ibídem. Rincón, p. 23.

14. Rueda L, Valdebenito C, Lolas F, Revista Chilena, artículo original. La Ocupación Ciencia y Técnica de Terapia Ocupacional para la intervención en psiquiatría, p. 33.

15. Soto N. Licenciatura en Terapia Ocupacional, disponible en teleton.org/ conocenos/universidad-teleton/licenciaturas-y-posgrados. Consultado el 31 de enero de 2013 a las 11:am. 